\title{
BMJ Open Effects of camera-based mirror visual feedback therapy for patients who had a stroke and the neural mechanisms involved: protocol of a multicentre randomised control study
}

\author{
Li Ding, ${ }^{1}$ Xu Wang, ${ }^{2}$ Xiaoli Guo, ${ }^{2}$ Shugeng Chen, ${ }^{1}$ Hewei Wang, ${ }^{1}$ Xiao Cui, ${ }^{3}$ \\ Jifeng Rong, ${ }^{4} \mathrm{Jie} \mathrm{Jia}{ }^{1}$
}

To cite: Ding L, Wang $X$, Guo $X$, et al. Effects of camerabased mirror visual feedback therapy for patients who had a stroke and the neural mechanisms involved: protocol of a multicentre randomised control study. BMJ Open 2019;9:e022828. doi:10.1136/ bmjopen-2018-022828

\section{- Prepublication history for} this paper is available online. To view these files, please visit the journal online (http://dx.doi. org/10.1136/bmjopen-2018022828).

Received 8 March 2018 Revised 25 December 2018 Accepted 2 January 2019

Check for updates

(C) Author(s) (or their employer(s)) 2019. Re-use permitted under CC BY-NC. No commercial re-use. See rights and permissions. Published by BMJ.

${ }^{1}$ Department of Rehabilitation Medicine, Huashan Hospital, Fudan University, Shanghai, China

${ }^{2}$ School of Biomedical Engineering, Shanghai Jiaotong University, Shanghai, China ${ }^{3}$ Department of Rehabilitation Medicine, Shanghai Changning Tianshan Traditional Medicine Hospital, Shanghai, China ${ }^{4}$ Department of Rehabilitation Medicine, The first Rehabilitation Hospital of Shanghai, Shanghai, China

Correspondence to

Dr Jie Jia; shannonjj@126.com

\section{ABSTRACT}

Introduction As a combination of visual stimulation and motor imagery, mirror visual feedback (MVF) is an effective treatment for motor impairment after stroke; however, few studies have investigated its effects on relevant cognitive processes such as visual perception and motor imagery. Camera-based MVF (camMVF) overcomes the intrinsic limitations of real mirrors and is recognised as an optimal setup. This study aims to investigate the effects of camMVF as an adjunct treatment for stroke patients, compare camMVF outcomes with those of conventional therapy and elucidate neural mechanisms through which MVF influences cognition and brain networks.

Methods and analysis This will be a multicentre, singleblinded, randomised controlled trial including 90 patients randomised into three groups: camera-based mirror visual feedback intervention group (30), shielded mirror visual feedback intervention group (30) and conventional group (30). Patients in each group will receive a $60 \mathrm{~min}$ intervention 5 days per week over 4 weeks. The primary outcome will be the Fugl-Meyer Assessment Upper Limb subscale measurement. Secondary outcomes include the modified Ashworth Scale, Grip Strength test, Modified Barthel Index, Functional Independence Measure, Berg Balance Scale, 10-metre walking test, hand-laterality task and electroencephalography .

Ethics and dissemination Ethics approval was granted by the Huashan Hospital Institutional Review Board on 15 March (KY2017-230). We plan to submit the results to a peer-reviewed journal and present them at conferences, rehabilitation forums and to the general public.

Trial registration number ChiCTR-INR-17013644; Preresults.

\section{INTRODUCTION}

Upper extremity motor impairment is a specific consequence of stroke. ${ }^{1}$ Approximately $65 \%$ of patients with hemisphere stroke live with paretic upper extremities, ${ }^{2}$ particularly the hands, which seriously affects motor performance and decreases the quality of life. Some evidence-based treatments such
Strengths and limitations of this study

- This is the first randomised controlled trial investigating the effect of camera-based motor imagery, mirror visual feedback (camMVF) on stroke patients and the underlying neural mechanisms.

- Our findings could help improve camMVF techniques and facilitate development of a novel MVF interface based on electroencephalography results.

- This study presents a method for developing a systematic procedure for mirror therapy.

- Future studies, including comparisons of camera and real-mirror-based MVF are required.

as constraint-induced movement therapy, robot-assisted therapy and mirror therapy (MT), among others, promote the recovery of the upper extremities and hands. ${ }^{3-5} \mathrm{MT}$, which is widely used in the rehabilitation of the upper extremities and hands, is less labour intensive and more convenient than other methods. ${ }^{6-8}$ In MT, a plane mirror is employed to provide a reflection of the movements of the unaffected hand. The reflection (referred to as mirror visual feedback or MVF) can generate a misperception of ownership which is recognised as a mirror illusion; however, the real mirror used in MT has some disadvantages including lack of balance control, postural pressure and weight shifting and it provides an undiversified training programme, all of which limit its clinical application. ${ }^{910}$ Numerous studies have proposed various technological approaches to create a new MVF interface to overcome these disadvantages. ${ }^{10-14}$ The feasibility of one such strategy for rehabilitation, camMVF, has been investigated in previous studies. 9131516 Our prior research demonstrated that camMVF can improve upper limb motor function and mental rotation ability in stroke patients. ${ }^{16}$ 
To optimise MT, a camMVF setup was employed in the present study to improve training posture, provide a more systematic training procedure and manipulable visual feedback. A previous report suggested that stroke patients with superior upper limb motor function have better balance control. ${ }^{17}$ Moreover, improved upper limb motor function may reduce the assistance required during transfer and ambulation, and elicit an interlimb reflex response, which can indirectly contribute to improvements in lower limb function. ${ }^{1718}$ Therefore, we hypothesise that camMVF can improve upper limb motor function in away similar to that of conventional MT and has the potential to improve the ability of patients to carry out daily activities, balance control and ambulation.

As a plasticity-based approach, the reversion of learnt non-use and activation of the central nervous system are the theoretical basis of MT. ${ }^{19-22}$ Compared with real mirrors, camMVF is, in theory, therapeutically identical. Electroencephalogram (EEG), functional MRI and functional near-infrared spectroscopy studies of amputees and healthy controls have suggested that camMVF can increase cortical activation of the sensorimotor cortex and the parietal and middle temporal cortices. ${ }^{10} 111523$ However, the effects of MVF on brain reorganisation in stroke patients remain unexplored. MVF is recognised as one component of graded motor imagery combined with visual stimulation. ${ }^{24-26}$ It is possible that MVF could promote the recovery of motor imagery ability, enhance visual perception of the affected limb and reorganise the corresponding brain network. Brain networks involved in motor imagery, particularly the extended motor network, are important for the motor processes that precede execution, such as motor preparation and planning. ${ }^{27-29}$ An abnormal extended motor network has even been found in stroke patients with good functional recovery and such abnormalities correlate with residual functional impairment. ${ }^{27}$ In our study, EEG recording, combined with a hand-laterality task which involves visual processing and mental rotation of hands, ${ }^{30}$ provides a good paradigm by which to study motor imagery and visual perception of the hands. Based on the results of our previous study, ${ }^{16}$ we hypothesised that improved brain network communication efficiency can contribute to performance in the hand-laterality task (reaction time and accuracy) following camMVF training intervention. Moreover, relying on network reorganisation, camMVF training can also lead to different manifestations of event-related potentials (ERPs).

\section{METHODS AND ANALYSIS Design}

This is a multicentre, single-blinded, randomised controlled trial (RCT)-(part of the camMVF study). A study flow diagram is presented in figure 1.

\section{Patient population}

Each centre is expected to randomise 30 stroke inpatients who meet the clinical criteria (table 1).

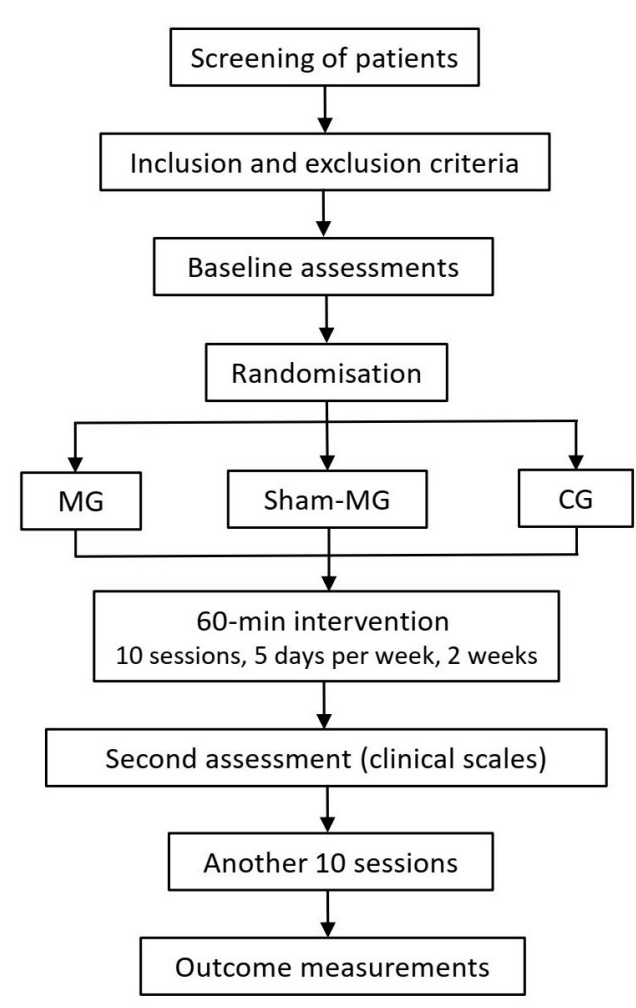

Figure 1 Trial flow chart. CG, conventional intervention group; MG, camera-based mirror visual feedback intervention group; Sham-MG, shielded mirror visual feedback intervention group.

\section{Randomisation}

Patients will be stratified based on motor deficit severity according to the Fugl-Meyer Upper Limb (FMA-UL) score, where patients with scores $\leq 35$ are classified as more impaired and those with scores $\geq 36$ as less impaired, ${ }^{31} 32$ and days from onset (early $<6$ months and late $\geq 6$ months). Eligible patients who are informed about and consent to participate in the study will receive a baseline assessment, and then be randomly allocated to one of the groups. Patients in each group will be treated separately, without

\begin{tabular}{|c|c|}
\hline Inclusion cr iteria & Exclusion criteria \\
\hline Age 25-75years & $\begin{array}{l}\text { Deteriorating medical } \\
\text { condition }\end{array}$ \\
\hline $\begin{array}{l}\text { Diagnosed with unilateral } \\
\text { stroke by CT or MRI between } \\
2 \text { weeks and } 1 \text { year following } \\
\text { stroke onset }\end{array}$ & $\begin{array}{l}\text { A history of epilepsy or } \\
\text { serious heart, lung, liver or } \\
\text { kidney function failure }\end{array}$ \\
\hline $\begin{array}{l}\text { Ability to follow the instructions } \\
\text { (MMSE } \geq 25 \text { ) }\end{array}$ & $\begin{array}{l}\text { Other problems that hinder } \\
\text { study implementation }\end{array}$ \\
\hline
\end{tabular}

Muscle tension (mAS $\leq 2)$

Ability to identify hand-laterality

mAS, modified Ashworth Scale; MMSE, mini-mental state examination. 
knowing their allocation during the entire study. Randomisation assignment will be generated using MATLAB (The MathWorks) by an independent researcher.

\section{Intervention}

Patients will be randomly assigned to one of three groups: camera-based mirror visual feedback intervention group (camMVF or MG), shielded mirror visual feedback intervention group (sham-MVF or sham-MG) or conventional intervention group (CG). The allocation sequence will be based on a computer-generated random number table. The randomisation programme and all assignments will be conducted by an independent researcher. During their hospitalisation, all inpatients will receive $60 \mathrm{~min}$ of treatment per day, for 5 days a week, lasting for 4 weeks (20 sessions). Hand-function rehabilitation $(30 \mathrm{~min}$ ) will be conducted for all patients following each treatment. Muscle stretch and massage will also be administered to patients before and after treatments for relaxation purposes, and all of these interventions will be in addition to their routine hospital treatments ( 2 hours per day).

\section{CamMVF intervention}

In this trial, we will use a camMVF box $(1200 \times 940$ $\times 702 \mathrm{~mm})$ to present manipulable visual feedback (mirrored, shielded, delayed and amplified) in place of a real plane mirror. Two mounted cameras will be used to capture hand motions and visual feedback will be presented using a 23.8-inch LED screen $(1920 \times 1080$ pixels). During treatment, patients will be seated in front of the LED screen at a comfortable height and place their hands in the box, which will block real visual feedback from both hands. The reflection and mirrored reflection of the unaffected hand will be presented on the screen, at a size similar size to that of real hands, during the $\mathrm{MG}$ intervention (figure 2). CamMVF provides a systematic procedure for MT, which contains basic and functional movement training items and verbal instructions with standard motion guide videos.

The basic part comprises 25 items that focus on the hand, wrist and forearm; these include grasp, finger-tofinger, wrist extension/flexion and forearm supination/ pronation. The functional part will include tool-based
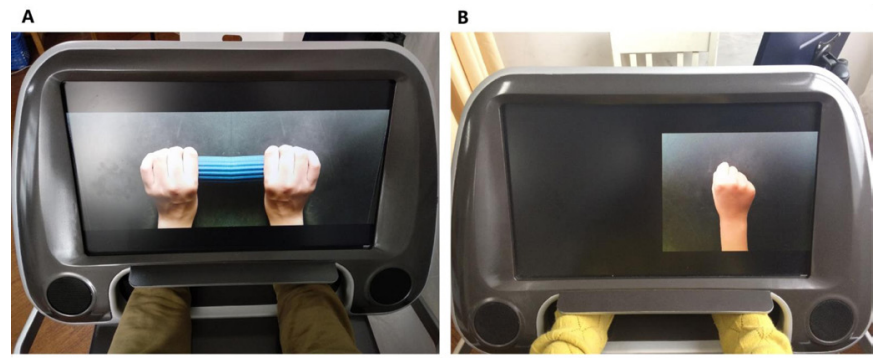

Figure 2 The camera-based mirror visual feedback (camMVF) system used in the present study. (A) Normal MVF of bar grasping for patients in the camMVF or MG group. (B) Shielded mirror visual feedback intervention group for making a fist for patients in the sham-MVF or sham-MG group. items such as bottle grasping and wooden cube picking. Therapists can choose any item to design a training plan according to the patient's motor impairments. Moreover, to make the training more self-guided and less labour intensive, there are verbal instructions/orders along with motion guiding videos during the initial training for whole treatment.

During the camMVF intervention, patients will be asked to carry out the training motions as symmetrically and synchronously as possible and to persuade themselves to imagine the moving hands on the screen are their own. An experienced therapist will design the training plan and adjust item difficulty to avoid global synkinesis of the affected limb and provide appropriate assistance. In this trial, every patient will receive a 60 -min training session, including 4-5 items (with 3-4 basic items and 1-2 functional items), and each item will be repeated 60 times per session.

\section{Sham-MVF intervention}

The camMVF box will also be used for the sham-MVF intervention; however, the reflection of the affected side will be shielded (figure 2). ${ }^{33}$ In the sham-MG group, patients will be required to perform the same exercises as those in the MG group, including the training protocol, intensity and duration. During training, patients will be required to attempt symmetrical movement and imagine that both hands are moving. We will compare the differences in clinical measurements and alterations in EEG signals before and after interventions between the two groups to explore the effects of MVF. ${ }^{34}$

\section{Conventional intervention}

Conventional intervention will comprise dosage-equivalent treatments of physiotherapy and/or occupational therapy focused on the hands, wrists and forearms (ie, the same exercise programmes without MVF). The training principle and items will be similar to those applied for the MG and sham-MG groups.

\section{Study outcomes}

The primary outcome and clinical assessments will be measured by an independent researcher at baseline and after 2 and 4 weeks of treatment. The hand-laterality task and EEG recording will be administered before and after the intervention by another researcher.

\section{Primary}

The FMA-UL subscale will be used to assess motor impairment as the primary outcome.

\section{Secondary}

\section{Clinical assessment}

Clinical measurements will include the modified Ashworth Scale, Grip Strength test,(hydraulic hand dynamometer, Exacta $^{\mathrm{TM}}$ ), modified Barthel Index, Functional Independence Measure, Berg Balance Scale, and 10 -metre walking test. These measurements focus on the evaluation of motor impairment, motor function, muscle 
tone and strength, hand dexterity (mild to moderately impaired patients), mobility and daily function.

\section{Hand-laterality task and EEG recording protocol}

The hand-laterality task is used to assess visual perception and motor imagery of the hands, and the reaction time and accuracy of the task will be measured. ${ }^{30}$ The patients will be seated in front of a laptop and asked to judge the laterality of hand images presented on the 13-inch display. The whole experiment consists of four blocks, following a single training block. There will be $3 \mathrm{~min}$ inter-block breaks. In each block, there will be 96 trials. In each trial, a black cross is displayed for $800 \mathrm{~ms}$ followed by stimulus images $(9 \times 9 \mathrm{~cm})$ of the back-view of the left or right hand at six different angles $\left(0^{\circ}, 60^{\circ}, 120^{\circ}, 180^{\circ}, 240^{\circ}\right.$ and $300^{\circ}$ ), giving a total of $2 \times 6$ types of stimulus images presented randomly with equal probability. Patients will be instructed to make hand-laterality judgements as quickly and accurately as possible by pressing a corresponding button using their unaffected hand. Images will be presented until the patient responds. Stimuli will be controlled using E-prime 2.0 (Psychology Software Tools, Pittsburgh, Pennsylvania, USA).

EEG signals will be collected from a 64-channel Ag/ AgCl EasyCap (Brain Products, Munich, Germany) and recorded during the hand-laterality task. All electrodes will be referenced to $\mathrm{FCz}$ and have impedance $<20 \mathrm{k}$. EEG signals will be amplified using a BrainAmp MR Plus amplifier (Brain Products GmbH, Munich, Germany) and recorded continuously using Vision Recorder (V.1.03) at a sampling rate of $1000 \mathrm{~Hz}$. ERPs and network properties (including clustering coefficient and characteristic path length) will be analysed and compared among groups to investigate the mechanism underlying camMVF.

\section{Statistical methods}

\section{Sample size}

We estimated the sample size required to detect differences in the effects of group $\times$ time interactions on clinical outcome (FMA-UL). An effect size (f) of 0.27 to 0.3 is expected based on previous MVF studies. ${ }^{11} 1635$ Given the expected effect size, a total sample size of 75 to 90 will be required for repeated analysis of variance (ANOVA) with a power of 0.8 and a two-sided type-I error of 0.01 . Therefore, we plan to recruit 90 patients (30 per group).

\section{Statistical analyses}

Primary analysis will be performed using the intentionto-treat principle. Treatment effects will be compared using a two-way repeated ANOVA for clinical measurements, taking TIME (three levels: before intervention and 2 and 4 weeks after intervention) as a within-subject factor and GROUP (three levels: MG, sham-MG and CG) as a between-subject factor. A three-way repeated ANOVA will be used to test behaviour during the hand-laterality task (response time and accuracy), taking TIME (two levels: before and after intervention) and HAND (two levels: affected and unaffected) as within-subject factors and GROUP (three levels: MG, sham-MG and CG) as a between-subject factor. A p value $<0.05$ will indicate statistical significance for all analyses.

\section{Patient and public involvement}

Development of the research question and intervention content was based on data from stroke patients in our previous pilot study who received MT via camMVF and achieved motor improvements. Training protocols were iteratively improved based on feedback from participants from July 2014. We assessed the participant burden of the intervention and research measures using group interviews and informal feedback in our previous pilot study. Patients will not be involved in participant recruitment or study conduct. We will send a summary of the study results to all participants.

\section{ETHICS AND DISSEMINATION}

This trial was registered on 2 December 2017 Patient recruitment began on 10 December 2017 and will continue until 31 December 2018. Primary data analysis began in October 2018. The institutional review board of Huashan Hospital will receive study reports at the middle and end of the study and monitor the study implementation and data collection. Any modifications to the protocol will also be agreed to by the review board. All study data will be preserved as case report forms. Huashan Hospital is the sponsor for the study. Patients will be recruited from Huashan Hospital Fudan University Jing'an Branch, the first Rehabilitation Hospital of Shanghai and Shanghai Changning Tianshan Traditional Medicine Hospital and receive interventions at these hospitals. This study protocol was written according to the Standard Protocol Items: Recommendations for Interventional Trials (SPIRIT) checklist. ${ }^{36}$ The study will eventually be published in a peer-reviewed journal, and the findings will be presented at conferences, rehabilitation forums and to the general public.

\section{DISCUSSION}

MT is a plasticity-based approach shown to have significant effects on motor impairment in $\mathrm{RCTs}^{367}$; however, real mirrors have some technological limitations and disadvantages, including weight shifting and postural pressure, ${ }^{9} 10$ which may be overcome using camMVF. The present study is aimed at testing the effectiveness of camMVF therapy, compare it with conventional treatment for stroke rehabilitation, and investigate the underlying neural mechanisms for involved aspects of cognition and brain networks. Our study will identify methods and systematic procedures for future implementation of the novel, manipulable camMVF method and facilitate better understanding of the central mechanisms involved in motor control which will improve MT effectiveness.

MVF is a visual stimulation combined with motor imagery. ${ }^{24-26}$ This special type of reflection can enhance 
the perception of affected limbs and increase the patient's sense of ownership. In addition, by activating the cognitive cortex, MVF can eventually activate the primary motor cortex and improve motor execution. ${ }^{37} 38$ Stroke disrupts both corticospinal output (eg, upstream motor execution) and motor processes (eg, attention, motor preparation and planning) ${ }^{29}$ Recognised as contributing to graded motor imagery, ${ }^{24}{ }^{26}$ camMVF may have the potential to improve motor imagery and visual perception of the affected hand, mediate motor cognitive processes, and eventually reorganise the motor network. According to the results of clinical measurements and EEG analysis of the MG, sham-MG and CG groups, the study aims to explore the neural mechanisms underlying camMVF and provide supplementary evidence of how this therapy can promote cortical reorganisation and plasticity.

Acknowledgements The authors would like to thank the participants of our previous pilot investigation for contributing to the study design by providing feedback about their experiences and preferences.

Contributors All the authors were involved in the conception and design of the research. $L D$ and XW are the principal investigators. XG will be responsible for EEG recording and analyses. SC and HW advised on the design of the camMVF system and treatment procedure. XC, JR, and JJ are responsible for the different study centres. JJ is the lead researcher and study manager. LD wrote the first draft, and all the authors contributed to the final version of this protocol.

Funding This work is supported by the National Key R\&D Program of China (2018YFC2002300 and 2018YFC2002301), the Science and Technology Commission of Shanghai Municipality (Grant No. 15441901602 and 16441905303), and the National Natural Science Foundation of China (Grant No. 61771313). The funding sources had no role in the design of this study and will not have any role during the study or publication.

Competing interests None declared.

Patient consent for publication Not required.

Ethics approval This study was approved by the Huashan Hospital Institutional Review Board on 15 March, 2017 (KY2017-230) in Shanghai, China.

Provenance and peer review Not commissioned; externally peer reviewed.

Open access This is an open access article distributed in accordance with the Creative Commons Attribution Non Commercial (CC BY-NC 4.0) license, which permits others to distribute, remix, adapt, build upon this work non-commercially, and license their derivative works on different terms, provided the original work is properly cited, appropriate credit is given, any changes made indicated, and the use is non-commercial. See: http://creativecommons.org/licenses/by-nc/4.0/.

\section{REFERENCES}

1. Pollock A, Farmer SE, Brady MC, et al. Interventions for improving upper limb function after stroke. Cochrane Database Syst Rev 2014;12:CD010820.

2. Dobkin BH. Rehabilitation after Stroke. N Engl J Med Overseas Ed 2005;352:1677-84

3. Thieme $\mathrm{H}$, Mehrholz J, Pohl M, et al. Mirror therapy for improving motor function after stroke. Stroke 2013;44:e1-2.

4. Lo AC, Guarino PD, Richards LG, et al. Robot-assisted therapy for long-term upper-limb impairment after stroke. N Engl J Med 2010;362:1772-83

5. Wolf SL, Winstein CJ, Miller JP, et al. Effect of constraint-induced movement therapy on upper extremity function 3 to 9 months after stroke. JAMA 2006;296:2095-104.

6. Wu CY, Huang PC, Chen YT, et al. Effects of mirror therapy on motor and sensory recovery in chronic stroke: a randomized controlled trial. Arch Phys Med Rehabil 2013;94:1023-30.

7. Samuelkamaleshkumar S, Reethajanetsureka S, Pauljebaraj P, et al. Mirror therapy enhances motor performance in the paretic upper limb after stroke: a pilot randomized controlled trial. Arch Phys Med Rehabil 2014;95:2000-5.
8. Hebert D, Lindsay MP, Mclntyre A, et al. Canadian stroke best practice recommendations: stroke rehabilitation practice guidelines, update 2015. Int J Stroke 2016;11:459-84.

9. Kim J, Yi J, Song CH. Kinematic analysis of head, trunk, and pelvic motion during mirror therapy for stroke patients. J Phys Ther Sci 2017;29:1793-9.

10. Mehnert J, Brunetti M, Steinbrink J, et al. Effect of a mirror-like illusion on activation in the precuneus assessed with functional nearinfrared spectroscopy. J Biomed Opt 2013;18:066001.

11. Giraux P, Sirigu A. Illusory movements of the paralyzed limb restore motor cortex activity. Neuroimage 2003;20:S107-11.

12. Sato K, Fukumori S, Matsusaki T, et al. Nonimmersive virtual reality mirror visual feedback therapy and its application for the treatment of complex regional pain syndrome: an open-label pilot study. Pain Med 2010;11:622-9.

13. Hoermann S, Ferreira Dos Santos L, Morkisch N, et al. Computerised mirror therapy with augmented reflection technology for early stroke rehabilitation: clinical feasibility and integration as an adjunct therapy. Disabil Rehabil 2017;39:1503-14.

14. Tyson S, Thomas N, Wilkinson J, et al. The effects of patient-directed mirror therapy on upper limb impairments and activity. Int $J$ Stroke 2014;9:16-17.

15. Lee HM, Li PC, Fan SC. Delayed mirror visual feedback presented using a novel mirror therapy system enhances cortical activation in healthy adults. J Neuroeng Rehabil 2015;12:56.

16. Ding L, Wang X, Guo X, et al. Camera-based mirror visual feedback: potential to improve motor preparation in stroke patients. IEEE Trans Neural Syst Rehabil Eng 2018;26:1897-905.

17. Stephenson JL, De Serres SJ, Lamontagne A. The effect of arm movements on the lower limb during gait after a stroke. Gait Posture 2010;31:109-15.

18. Lin KC, Huang PC, Chen YT, et al. Combining afferent stimulation and mirror therapy for rehabilitating motor function, motor control, ambulation, and daily functions after stroke. Neurorehabil Neural Repair 2014;28:153-62.

19. Dohle C, Püllen J, Nakaten A, et al. Mirror therapy promotes recovery from severe hemiparesis: a randomized controlled trial. Neurorehabil Neural Repair 2009;23:209-17.

20. Garrison KA, Winstein CJ, Aziz-Zadeh L. The mirror neuron system: a neural substrate for methods in stroke rehabilitation. Neurorehabil Neural Repair 2010;24:404-12.

21. Nojima I, Mima T, Koganemaru S, et al. Human motor plasticity induced by mirror visual feedback. J Neurosci 2012;32:1293-300.

22. Deconinck FJ, Smorenburg AR, Benham A, et al. Reflections on mirror therapy: a systematic review of the effect of mirror visual feedback on the brain. Neurorehabil Neural Repair 2015;29:349-61.

23. Franz EA, Fu Y, Moore M, et al. Fooling the brain by mirroring the hand: brain correlates of the perceptual capture of limb ownership. Restor Neurol Neurosci 2016;34:721-32.

24. Polli A, Moseley GL, Gioia E, et al. Graded motor imagery for patients with stroke: a non-randomized controlled trial of a new approach. Eur J Phys Rehabil Med 2017;53:14-23.

25. Johnson S, Hall J, Barnett $\mathrm{S}$, et al. Using graded motor imagery for complex regional pain syndrome in clinical practice: failure to improve pain. Eur J Pain 2012;16:550-61.

26. Moseley GL. Graded motor imagery for pathologic pain: a randomized controlled trial. Neurology 2006;67:2129-34.

27. Sharma N, Baron JC, Rowe JB. Motor imagery after stroke: relating outcome to motor network connectivity. Ann Neurol 2009;66:604-16.

28. Yan J, Guo X, Jin Z, et al. Cognitive alterations in motor imagery process after left hemispheric ischemic stroke. PLoS One 2012;7:e42922.

29. Sharma N, Simmons LH, Jones PS, et al. Motor imagery after subcortical stroke: a functional magnetic resonance imaging study. Stroke 2009;40:1315-24.

30. Guo X, Lin Z, Lyu Y, et al. The effect of prosthesis use on hand mental rotation after unilateral upper-limb amputation. IEEE Trans Neural Syst Rehabil Eng 2017;25:2046-53.

31. Winstein CJ, Wolf SL, Dromerick AW, et al. Interdisciplinary Comprehensive Arm Rehabilitation Evaluation (ICARE): a randomized controlled trial protocol. BMC Neurol 2013;13:5.

32. Fugl-Meyer AR, Jääskö L, Leyman I, et al. The post-stroke hemiplegic patient. 1. a method for evaluation of physical performance. Scand J Rehabil Med 1975;7:13-31.

33. Cacchio A, De Blasis E, Necozione S, et al. Mirror therapy for chronic complex regional pain syndrome type 1 and stroke. N Engl J Med 2009;361:634-6.

34. Ng MJ, Singh P, Pandian JD, et al. Mirror therapy in unilateral neglect after stroke (MUST trial): a randomized controlled trial. Neurology 2015;84:1286.

35. Lee $\mathrm{D}$, Lee $\mathrm{M}$, Lee $\mathrm{K}$, et al. Asymmetric training using virtual reality reflection equipment and the enhancement of upper limb function in 
stroke patients: a randomized controlled trial. J Stroke Cerebrovasc Dis 2014;23:1319-26.

36. Chan AW, Tetzlaff JM, Altman DG, et al. SPIRIT 2013 statement: defining standard protocol items for clinical trials. Ann Intern Med 2013;158:200-7.
37. Carson RG, Ruddy KL. Vision modulates corticospinal suppression in a functionally specific manner during movement of the opposite limb. J Neurosci 2012;32:646-52.

38. Dohle C, Stephan KM, Valvoda JT, et al. Representation of virtual arm movements in precuneus. Exp Brain Res 2011;208:543-55. 